\section{Carbon dioxide enrichment restrains the impact of drought on three maize hybrids differing in water stress tolerance in water stressed environments}

\author{
Jinyoung Yang, ${ }^{1}$ \\ Richard C. Sicher, ${ }^{1}$ Moon S. Kim, ${ }^{2}$ \\ Vangimalla R. Reddy ${ }^{1}$ \\ ${ }^{1}$ Crop Systems and Global Change \\ Laboratory, ${ }^{2}$ Environmental Microbial and \\ Food Safety Laboratory, USDA-ARS, \\ Beltsville, MD, USA
}

\section{Abstract}

Three maize genotypes were grown in controlled environment chambers with ambient (38 $\mathrm{Pa})$ or elevated $(70 \mathrm{~Pa})$ carbon dioxide and water stress treatments were initiated 17 days after sowing. Shoot dry weight of the drought tolerant hybrid in both $\mathrm{CO}_{2}$ treatments was 44 to $73 \%$ less than that of the intermediate and sensitive hybrids when seedlings were well watered. Decreased shoot and root dry weights of the tolerant maize hybrid due to drought were about one-half that of the other two hybrids. Genotypic differences were observed in decreases of soil water content, leaf water potential, net photosynthesis and stomatal conductance in response to drought. Eleven of 19 amino acids measured in this study increased, methionine was unchanged and alanine and aspartate decreased in response to drought in the ambient $\mathrm{CO}_{2}$ treatment. Increased amino acid levels under elevated $\mathrm{CO}_{2}$ were observed at the end of the experiment. Significant genotypic differences were detected for amino acid responses to drought. Effects of drought on all three genotypes were mitigated by $\mathrm{CO}_{2}$ enrichment. Decreased shoot growth likely improved the stress tolerance of a highly drought resistant maize hybrid by reducing moisture loss, improving soil moisture content and increasing leaf water potential.

\section{Introduction}

The production of maize is of global importance because of its high yield potential, its many industrial uses and its suitability as an animal feedstock. ${ }^{1}$ Most maize growing areas are rain-fed and the crop is subject to periodic water deficits that diminish yields. ${ }^{2}$ Soil water deficiencies can result from insufficient rain- fall or from the degradation of natural groundwater sources. Global climate change is predicted to increase ambient temperatures and also the frequency and severity of drought in various growing regions that are highly dependent on maize. . $^{1,3}$ To improve production in water limited environments major seed companies have developed maize germplasm with enhanced yields under moisture stress. Both Syngenta (Basel, Switzerland) and Pioneer Hi-Bred International (Johnston, IA, USA) are marketing drought tolerant germplasm (AquasureArtesian $^{\mathrm{TM}}$ and Optimum AQUAmax ${ }^{\mathrm{TM}}$, respectively) in the United States that reportedly improved maize yields under water limited conditions by $15 \%$ and $5 \%$, respectively. ${ }^{4}$ This germplasm is marketed to farmers growing maize in the semiarid, western areas of the North American Corn Belt and, importantly, yields under water sufficient conditions are only marginally less than control hybrids. The International Maize and Wheat Improvement Center (CIMMYT) and the International Institute for Tropical Agriculture (IITA) are jointly developing drought resistant maize varieties for release in Sub-Saharan Africa that could potentially increase maize yields up to $34 \%{ }^{5}$ Phenotypic and metabolic drought responses of CIMMYT derived maize hybrids differing in drought tolerance and developed for use in Africa have been described previously. ${ }^{6,7}$ Also, Monsanto received approval to market a drought tolerant, transgenic maize hybrid (MON 87460) that expresses a stress-related bacterial RNA chaperone, known as cold shock protein B. ${ }^{8}$ Taken together, maize germplasm with enhanced desiccation tolerance could be an important tool for analyzing and understanding plant responses to environmental stress.

Drought resistant maize genotypes are normally developed by applying water stress during reproductive growth and by quantifying phenotypic traits, such as silking, yield, grain number, carbon allocation to roots, leaf rolling and leaf chlorophyll content. ${ }^{7}$ Successful stress resistant genotypes were ultimately selected on the basis of yield in moisture depleted environments. How the above mentioned drought resistant maize germplasm responds to water stress during early, vegetative growth is less well documented. We recently reported the effects of $\mathrm{CO}_{2}$ enrichment on primary metabolism in maize leaves using control and water stressed vegetative plants. ${ }^{9}$ In this earlier study about $85 \%$ of the major metabolites in maize leaves were impacted by drought and about one-fourth of the affected metabolites responded differently when drought treatments were imposed under $\mathrm{CO}_{2}$ enrichment. The primary effect of elevated $\mathrm{CO}_{2}$ treatments was to delay the impact of soil moisture deficits on maize by decreasing rates of stom-
Correspondence: Jinyoung Yang, USDA-ARS, Crop Systems and Global Change Laboratory, 10300 Baltimore Avenue, Building 001, Room 342, BARC-WEST, Beltsville, MD 20705, USA Tel. +1.301.504.5686 - Fax:+1.301.504.5826. E-mail: jinyoung.barnaby@ars.usda.gov

Key words: plant stress, biomass allocation, drought tolerance, maize genotypes, amino acid metabolism, proline accumulation.

Acknowledgments: the authors would thank Robert Erdman, Fran Caulfield and Alex Song for valuable technical assistance. Dr. James Bunce provided several helpful suggestions during preparation of the manuscript.

Contributions: JYB, data collecting and analyzing; JYB and RCS, manuscript writing; MSK and VRR manuscript reviewing and references search.

Conflict of interests: the author declare no potential conflict of interests.

Funding: the work was supported by USDA (CRIS\# 1265-11210-008-D).

Revision received: 30 June 2014.

Accepted for publication: 2 August 2014.

This work is licensed under a Creative Commons Attribution NonCommercial 3.0 License (CC BYNC 3.0).

(C)Copyright J.Y. Barnabyet al., 2014 Licensee PAGEPress srl, Italy

International Journal of Plant Biology 2014; 5:5535 doi:10.4081/pb.2014.5535

atal conductance. Changes of specific metabolites, such as proline and malate, were sensitive indicators of drought in maize leaves and responses of these compounds to water stress varied markedly under ambient and elevated $\mathrm{CO}_{2}$ growth conditions. Witt et al. ${ }^{6}$ previously studied six maize hybrids differing in resistance to water stress and concluded that changes of metabolite markers in five different maize tissues were not correlated with drought tolerance. This is an important question that we would like to revisit.

The objective of the current study was to compare responses to water stress during vegetative growth of Optimum AQUAmax ${ }^{\mathrm{TM}}$ (Pioneer Hi-Bred International, USA) lines and that of an elite control maize hybrid line also from Pioneer-Hi Bred International with differing moisture stress tolerance. We hypothesized that stomatal function, leaf water relations, photosynthesis and leaf metabolite levels would differ between drought resistant and drought susceptible maize genotypes and that $\mathrm{CO}_{2}$ enrichment would modify genotypic responses to water stress. 


\section{Materials and Methods}

\section{Plant materials}

Elite hybrid maize germplasm (Zea mays L.) with differing drought sensitivity was obtained from Jerron Schmoll, Technical Services Manager, Pioneer Hi-Bred International, Inc., Columbus, $\mathrm{OH}$, USA. The maize hybrids used in this study were 33P84 (drought susceptible control), P0791HR (intermediate drought tolerance) and P1151HR (drought tolerant). These are hereafter referred to as the susceptible, intermediate and tolerant maize hybrids, respectively. The two drought resistant maize lines were marketed in 2011 as 0ptimum AQUAmax hybrids. All three hybrid lines in this study are transgenic and contain multiple foreign genes that confer resistance to various herbicides and plant pests. However, resistance to water stress was obtained from native drought tolerance genes obtained by marker specific selection and enhanced breeding techniques. Maize seedlings were grown in a matching pair of controlled environment chambers (model M-2, Environmental Growth Chamber Corp., Chagrin Falls, OH, USA) essentially as described previously. ${ }^{9}$ Prior to planting, seeds of all three hybrid lines were imbibed over night on wetted filter paper, soaked for $15 \mathrm{~min}$ in onethird strength commercial bleach and were washed thoroughly with sterile, deionized water. After surface sterilization multiple seeds were planted in $1.8 \mathrm{dm}^{3}$ plastic pots filled with vermiculite. The air temperature was $27 \pm 1^{\circ} \mathrm{C}$, the PPFD was $700 \pm 40 \mu \mathrm{mol} \mathrm{m}{ }^{-2} \mathrm{~s}^{-1}$, when measured at pot height, and ambient and elevated chamber air $\mathrm{CO}_{2}$ partial pressures were $38 \pm 10$ $\mathrm{Pa}$ and $70 \pm 10 \mathrm{~Pa}$, respectively. Plants were grown with a $14 \mathrm{~h}$ day/10h night cycle and pots were watered to the drip point once daily with a complete mineral nutrient solution containing $14.5 \mathrm{mM}$ total N. Normally, seeds were sown in eight pots per treatment and these were thinned to one plant per pot between 5 and 7 DAS. One chamber in each pair was randomly chosen for drought treatment which was imposed by withholding nutrient solution. Chambers chosen to receive water stress treatments were alternated between plantings. Water stress treatments were initiated 17 DAS and were continued for up to 17 and $19 \mathrm{~d}$ in the ambient and the elevated $\mathrm{CO}_{2}$ treatments, respectively. Relative humidity was not controlled in these experiments but $24 \mathrm{~h}$ averages were $65 \pm 10 \%$ prior to initiating drought treatments. Leaf sections having 5 to $10 \mathrm{~cm}^{2}$ in area were removed from the midpoint of the most recent, fully expanded leaf, i.e., normally the $4^{\text {th }}$ leaf from emergence. Samples were harvested between 4 and $6 \mathrm{~h}$ after the start of the photoperiod on indicated days using four plants from each $\mathrm{CO}_{2}$ and drought treatment. Leaf samples were quickly transferred to small envelopes, placed in liquid $\mathrm{N}_{2}$ to quench metabolism and stored at $-80^{\circ} \mathrm{C}$ for up to 1 month prior to analysis without altering the results.

\section{Amino acid measurements}

Maize leaf soluble amino acids were measured using an Ultra-Performance Liquid Chromatography procedure as described previously. ${ }^{9} \mathrm{~A} 1 \mathrm{~mL}$ aliquot of an aqueous-methanol extract was evaporated to dryness under a stream of $\mathrm{N}_{2}$ at $37^{\circ} \mathrm{C}$. The dried aliquot was resuspended in $0.02 \mathrm{~N} \mathrm{HCl}$, filtered through a $0.22 \mu \mathrm{m}$ spin filter and derivative with an Accq Tag Ultra kit for amino acid analysis (No. 186003836, Waters Corp., Milford, MA, USA). Standard curves were prepared with a known mixture of 19 amino acids for each sample set. Except for glutamine and arginine the amino acid standards, plus ammonia and the internal standard were completely separated by using the method described above.

\section{Gas exchange measurements}

Gas exchange parameters were measured during the middle of the light period using the same intact maize leaves that were used for metabolite analyses. ${ }^{9}$ Measurements were initiated 17 DAS and were performed at 1 or $2 \mathrm{~d}$ intervals throughout the drought treatment. Net $\mathrm{CO}_{2}$ and $\mathrm{H}_{2} \mathrm{O}$ vapor exchange rates were determined with a model 6400 portable Photosynthesis System (Open System 4.0, LiCor, Inc., Lincoln, NE, USA). Individual leaves from a single maize plant were placed in a 1 $\mathrm{dm}^{3}$ cuvette and conditions within the cuvette were set to match that of the growth chambers used for plant growth. Initially, the PPFD, temperature and relative humidity in the cuvette were $700 \pm 40$ (chamber condition) $\mu \mathrm{mol} \mathrm{m}{ }^{-2} \mathrm{~s}^{-}$ ${ }^{1}, 27^{\circ} \mathrm{C}$ and $\sim 70 \%$, respectively. Illumination in the cuvette was from a red/blue LED lamp provided by the instrument's manufacturer. The $\mathrm{CO}_{2}$ partial pressure within the cuvette was either $38 \mathrm{~Pa}$ or $68 \mathrm{~Pa} \mathrm{CO}_{2}$ and temperature did not vary during measurement. Leakage rates during gas-exchange measurements were determined with an empty cuvette. Gas exchange data were collected using three to five plants from each drought treatment per experiment. Values of PN, Ci and gs were calculated by the Photosynthesis System.

\section{Other methods}

Immediately after completing the gasexchange analyses $6 \mathrm{~mm}$ diameter leaf discs were removed from the measured leaves and placed in insulated ceramic cups. Leaf water potential was determined with a model HR-33T dewpoint microvoltmeter after a $1 \mathrm{~h}$ incubation period (Wescor, Logan UT, USA). Relative changes of soil water content were determined by measuring pot weights at indicated times and comparing this to pots containing either completely saturated or dried potting material. For biomass determinations plants were separated into root and shoot fractions and these were oven dried at $70^{\circ} \mathrm{C}$ for $72 \mathrm{~h}$ prior to weighing.

\section{Statistical comparisons}

Results of two completely replicated experiments for each $\mathrm{CO}_{2}$ treatment were combined and significant differences were determined using a two-way Analysis of Variance procedure (StatView, Mountain View, CA). Leaf components were the independent variables and drought, $\mathrm{CO}_{2}$ and date were the dependent variables. Ratios were obtained by dividing the drought treated values with corresponding values for the water sufficient controls and the amino acid data in this study were used for hierarchical cluster analysis as described by Suzuki et al. ${ }^{10}$ Additional details were described previously. ${ }^{9}$

\section{Results}

Genotypic differences of dry matter allocation in seedlings of maize hybrids impacted by water stress and $\mathrm{CO}_{2}$ enrichment

Biomass formation of three maize genotypes differing in drought tolerance was determined following 15 and $17 \mathrm{~d}$ of drought treatment in the ambient and elevated $\mathrm{CO}_{2}$ environments, respectively (Figure 1A,B). Both shoot and root $\mathrm{DW}$ of the intermediate andsensitive maize hybrid lines were similar $(\mathrm{P} \geq 0.05)$ in the water sufficient and insufficient treatments of both $\mathrm{CO}_{2}$ environments. Because total days of water stress treatment differed, final DW values were not directly comparable between the ambient and elevated $\mathrm{CO}_{2}$ treatments. When seedlings were well watered, shoot DW of the drought tolerant cultivar in both $\mathrm{CO}_{2}$ treatments was 44 to $73 \%$ less than that of the intermediate and sensitive maize hybrid lines. Similar changes were observed for root DW. Dry matter accumulation in shoots of the intermediate and sensitive maize hybrid lines decreased between 61 and $73 \%$ in response to the water stress treatments in both $\mathrm{CO}_{2}$ environments. In comparison, shoot DW of the tolerant maize hybrid line decreased $32.5 \%$ on average in response to water stress when the results of both $\mathrm{CO}_{2}$ treatments were combined. Root DW of the intermediate and sensitive maize lines also decreased between 17 and $33 \%$ in response to drought in both $\mathrm{CO}_{2}$ environments. Conversely, root DW of the tolerant maize hybrid line increased between 5 and $26 \%$, respectively, in the water depleted ambient and elevated $\mathrm{CO}_{2}$ treatments. 
Genotypic differences of single leaf gas exchange rates of maize hybrids impacted by water stress and $\mathrm{CO}_{2}$ enrichment (stomatal density)

Measured values of SWC after $0 \mathrm{~d}$ of drought treatment were near $100 \%$ under well watered conditions and these SWC values increased on subsequent harvest dates (Figure 2A-C). For all three genotypes in the ambient $\mathrm{CO}_{2}$ treatment, SWC decreased slowly for the first $3 \mathrm{~d}$ of water stress and then SWC decreased in a near linear manner for the next 7 or $8 \mathrm{~d}$. Finally, the rate of change in SWC slowed toward the end of the experiment. The slowdown in water consumption after 10 or $11 \mathrm{~d}$ of water stress was not observed in the elevated C02 treatment for any of the genotypes used in this study. Final SWC values in the ambient $\mathrm{CO}_{2}$ treatment were $14.0 \pm 0.7,16.1 \pm 1.9$ and $26.3 \pm 3.5 \%$ for the sensitive, intermediate and tolerant maize hybrid lines, respectively. Comparable values in the elevated $\mathrm{CO}_{2}$ treatment were $15 \pm 2.0$, $18.6 \pm 3.1$ and $25.9 \pm 2.5 \%$. These measurements were performed in the ambient and elevated $\mathrm{CO}_{2}$ treatments after 15 and $17 \mathrm{~d}$, respectively, of drought treatment. Therefore, the net effect of $\mathrm{CO}_{2}$ enrichment was to decrease the rate of water uptake and to lengthen the experiment by $2 \mathrm{~d}$. Changes of LWP in the sensitive, intermediate and drought tolerant maize lines were in close agreement with responses of PN to water stress and $\mathrm{CO}_{2}$ enrichment as discussed below. For all three maize lines, initial LWP values were about $-0.8 \mathrm{MPa}$ and little or no changes occurred in the well-watered control leaves throughout the experiment (Figure 2DF). The lowest LWP values $(-1.9 \mathrm{MPa})$ were observed on the final harvest for the sensitive maize line grown under ambient $\mathrm{CO}_{2}$. Changes of LWP in response to drought were greatest for the sensitive line and least for the drought tolerant line. Again, $\mathrm{CO}_{2}$ enrichment delayed the impact of water stress on LWP by about $2 \mathrm{~d}$.

Water stress and CO2 enrichment impacted $g_{s}$ of maize leaves (Figure 2G-I). Rates of $g_{s}$ for the water-sufficient tolerant, intermediate and sensitive maize hybrid lines were about 0.2 mmol m${ }^{-2} \mathrm{~s}^{-1}$ in the ambient $\mathrm{CO}_{2}$ treatment and values were essentially unchanged over the duration of the experiment. Values of $g_{s}$ decreased 50 to $60 \%$ when maize seedlings were grown in elevated compared to ambient $\mathrm{CO}_{2}$ using well watered treatment conditions. Near linear decreases of $g_{s}$ were observed in response to water stress treatments when all three maize genotypes were grown under ambient $\mathrm{CO}_{2}$. Relative decreases of $g_{s}$ under ambient $\mathrm{CO}_{2}$ were greater for the sensitive and intermediate genotypes than for the drought tolerant line. Relative changes of $g_{s}$ in response to drought for all three genotypes were smaller under elevated than under the ambient $\mathrm{CO}_{2}$ treatment. Note also that rates of $g_{s}$ for the sensitive and intermediate hybrids in response to drought were similar in both $\mathrm{CO}_{2}$ environments on the last measurement dates. This was not true for the drought tolerant genotype, where absolute changes of $g_{s}$ in response to water stress were smaller than for the other two maize hybrids.

Mean rates of $\mathrm{P}_{\mathrm{N}}$, measured prior to initiating water stress and averaged over both $\mathrm{CO}_{2}$ treatments, were $19.3 \pm 1.4,17.6 \pm 1.1$ and $20.6 \pm 0.8 \mu \mathrm{mol} \mathrm{m}^{-2} \mathrm{~s}^{-1}$ for the tolerant, intermediate and susceptible maize lines, respectively (Figure 2J-L). Single leaf $\mathrm{CO}_{2}$ assimilation rates of well watered control plants of all three hybrid lines from both $\mathrm{CO}_{2}$ treatments increased up to $20 \%$ over the course of the experiment. Rates of PN by the sensitive line decreased $95 \%$ and $84 \%$ in the ambient and elevated $\mathrm{CO}_{2}$ treatments, respectively, when measured between 15 and $17 \mathrm{~d}$ of drought. Rates of PN by the sensitive hybrid line under ambient $\mathrm{CO}_{2}$ decreased incrementally over the first few measurement dates and then the inhibition of $\mathrm{P}_{\mathrm{N}}$ became more severe as the extent of drought worsened. Changes of $P_{N}$ for the sensitive maize line grown with elevated $\mathrm{CO}_{2}$ were minimal for the first $9 \mathrm{~d}$ of drought treatment but on subsequent measurement dates photosynthetic capacity decreased rapidly. Observed rates of $\mathrm{P}_{\mathrm{N}}$ by the intermediate maize line also decreased over $90 \%$ in response to $15 \mathrm{~d}$ of water insufficiency in the ambient $\mathrm{CO}_{2}$ treatment. Unlike the sensitive line, changes of $\mathrm{P}_{\mathrm{N}}$ in response to drought were minimal over the first $9 \mathrm{~d}$ of drought treatment in both $\mathrm{CO}_{2}$ environments. When the intermediate maize line was grown in elevated $\mathrm{CO}_{2}$, rates of $\mathrm{P}_{\mathrm{N}}$ decreased $60 \%$ due to water stress between the initial and final

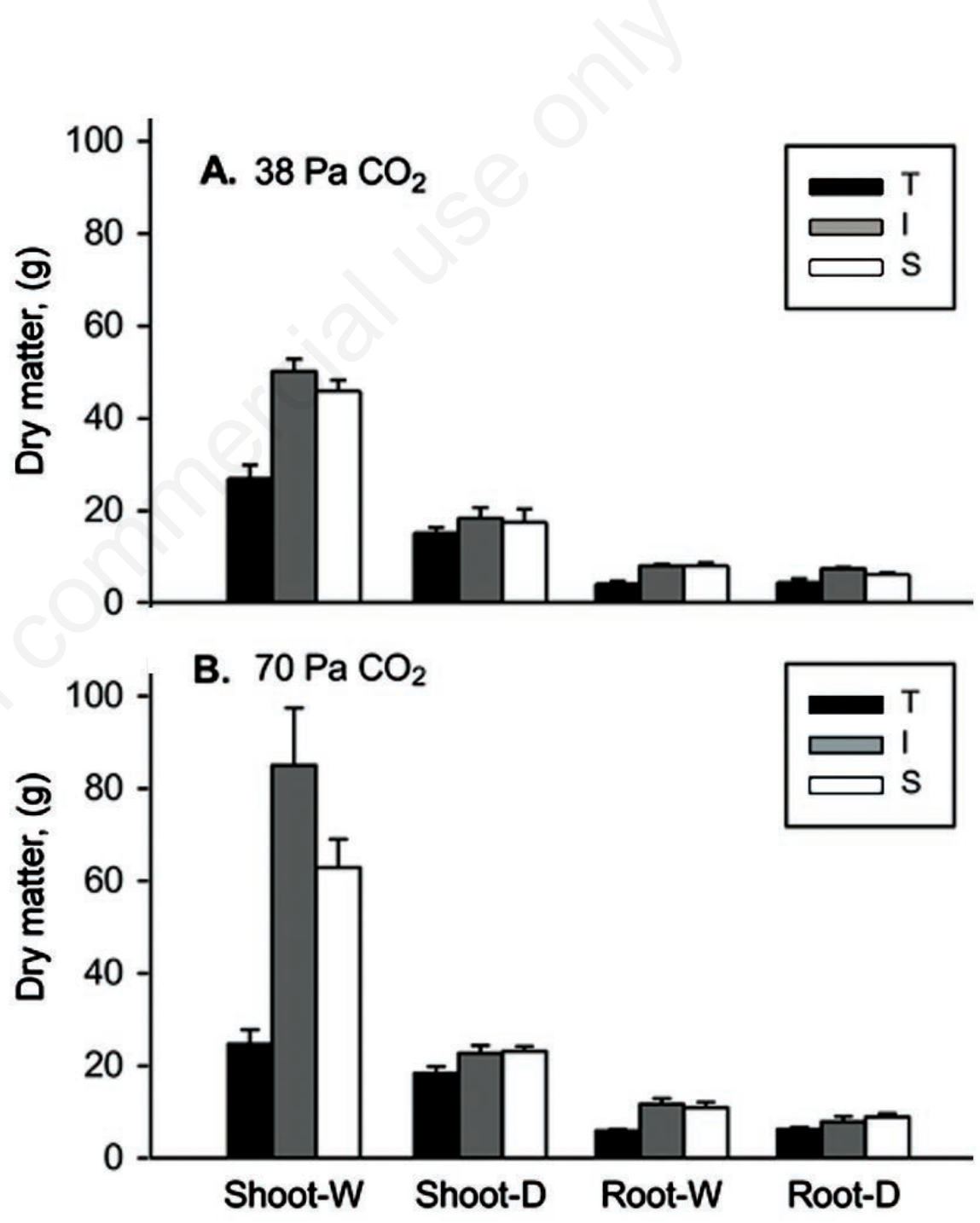

Figure 1. Dry matter accumulation of three maize hybrids differing in drought resistance. Drought treatments were applied to $17 \mathrm{~d}$ old sensitive (gray fill), intermediate (black fill) and drought tolerant (light gray fill) maize hybrids. Shoot (left two columns) and root (right two columns) dry matter (g) was measured after 17 (ambient, Panel A) or 19 (elevated, Panel B) $d$ of drought treatment. Values are means \pm standard error for eight samples from two fully replicated experiments 
measurement dates. Irrespective of chamber air $\mathrm{CO}_{2}$ levels, the drought tolerant maize line retained $89 \%$ or more of initial $\mathrm{P}_{\mathrm{N}}$ rates when water was withheld for 15 to 17.

Effects of water stress and $\mathrm{CO}_{2}$ enrichment on select soluble amino acid in leaves of maize hybrids differing in drought tolerance

Effects of genotype and $\mathrm{CO}_{2}$ enrichment on changes of nine amino acids in maize leaves in response to water stress are shown in Figure 3 . These nine amino acids displayed the largest and most consistent responses to drought in this study. Asp and Ala are major components of maize leaves and these amino acids decreased over $90 \%$ in all three genotypes and in either $\mathrm{CO}_{2}$ treatment in response to water stress. In leaves of well watered control plants, Asp increased about 2 -fold between the first and last harvest and this was true for both $\mathrm{CO}_{2}$ treatments and all three genotypes. Ala levels were variable in well watered control plants from the ambient $\mathrm{CO}_{2}$ treatment. In the elevated $\mathrm{CO}_{2}$ treatment, Ala levels in water sufficient control plants of all three maize hybrids were 50 to $51 \%$ lower on the final harvest when compared to initial values. Asp and Ala were little changed by water insufficiency over the first $10 \mathrm{~d}$ of drought treatment when plants were grown in elevated $\mathrm{CO}_{2}$ but levels of these two amino acids decreased in response to water stress on subsequent harvests when compared to water sufficient maize hybrids.

Levels and responses of Glu in leaves of water sufficient plants of all three maize genotypes and in both the ambient and elevated $\mathrm{CO}_{2}$ treatments were similar to results observed for Asp. This was also true for Glu measurements of water deficient plants of all three maize genotypes grown under elevated $\mathrm{CO}_{2}$. However, Glu levels in water depleted leaves of the sensitive and intermediate maize lines did not increase initially in response to drought in the ambient $\mathrm{CO}_{2}$ treatment. Subsequently, Glu in these two maize lines increased and attained control levels when the leaf water potential became more negative. Effects of the water stress on Glu levels under ambient $\mathrm{CO}_{2}$ were less in the tolerant compared to the intermediate and sensitive hybrid maize lines. Under elevated $\mathrm{CO}_{2}$, Glu levels decreased in all three genotypes in response to water stress.

The other six soluble amino acids reported in this study increased in response to water stress and this effect was greater under ambient than under elevated $\mathrm{CO}_{2}$. In the current study, Pro levels in the sensitive and intermediate hybrid lines increased between 120 and 335 fold on a DW basis in response to drought when grown in ambient $\mathrm{CO}_{2}$. In comparison, Pro in the intermediate and tolerant maize lines was unaffected by drought when experiments were performed under elevated $\mathrm{CO}_{2}$. None of the amino acids measured in this study increased with drought in leaves of the tolerant line when grown in $\mathrm{CO}_{2}$ enriched atmospheres. However, Pro levels in the sensitive line increased 85 fold on the penultimate harvest when grown under elevated $\mathrm{CO}_{2}$. Five other amino acids in this study increased in response to water stress in the elevated $\mathrm{CO}_{2}$ treatment but this was only observed after $17 \mathrm{~d}$ of drought treatment.

Changes of Asn and Phe in all three genotypes and both $\mathrm{CO}_{2}$ treatments in response to water stress were broadly similar to that for Pro. However, the responses of Leu, Ile and Tyr to drought differed from that of the other amino acids reported here in that maximum levels of these three amino acids were greater in the tolerant than in the intermediate or sensitive genotype. The other amino acids measured here, i.e., Gly, Ser, His, Val, Thr, Lys and Cys, were less affected by drought in this study than the nine soluble amino acids shown in Figure 3 (data not shown).

Hierarchical clustering was used to identify associations between the nine amino acids shown in Figure 3 and drought responses of the three maize genotypes used in this study (Figure 4). Three separate metabolite clusters were identified and these differed significantly $(\mathrm{P} \leq 0.05)$. The first cluster was for amino acids

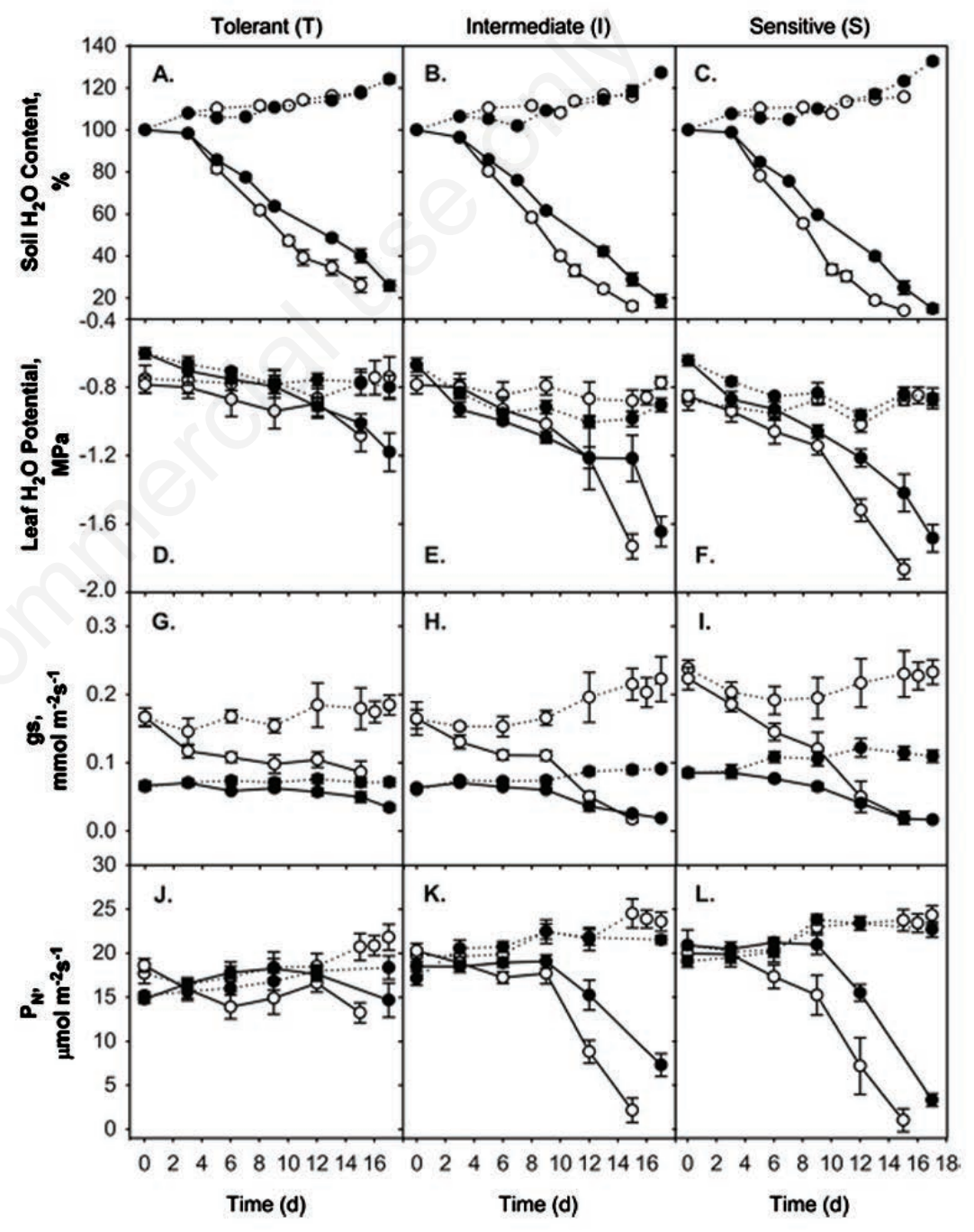

Figure 2. Genotypic differences of water relations and of single leaf gas exchange among three maize hybrids differing in susceptibility to drought. Changes of net photosynthesis ( $\mu \mathrm{mol} \mathrm{m} \mathrm{m}^{-2} \mathrm{~s}^{-1}$; A-C), stomatal conductance (mmol m-2s-1; D-F), leaf water potential (MPa, G-I) and soil water content (\%: J-L) in response to drought are shown for the intermediate $(A, D, G, J)$, tolerant $(B, E, H, K)$ and sensitive $(C, F, I, L)$ maize hybrids. Results are shown for water sufficient (dotted line), water insufficient (solid line) ambient $(O)$ and elevated $(\bullet) \mathrm{CO}_{2}$ treatments. Values are means \pm standard error for eight samples from two fully replicated experiments. 
of all three genotypes that either decreased or failed to increase in response to drought in both $\mathrm{CO}_{2}$ environments. Amino acids found in the first cluster were Ala, Asp and Glu and these were from leaves of all three maize hybrids. The first cluster also contained Pro and Asn of the drought resistant genotype. The second cluster contained amino acids that increased modestly in response to drought and the amino acids affected in this manner were Ile, Tyr, Leu and Phe. The second cluster only contained amino acids from the drought tolerant hybrid. The third metabolite cluster contained amino acids that increased the most in this study in response to drought. Cluster three only contained amino acids from the intermediate and sensitive maize hybrids. The clustering analysis clearly showed genotypic differences in amino acid changes to drought. The largest genotypic differences in this study were for Pro in Asn. The heat map image in Figure 4 also confirmed that changes of amino acids in the three maize hybrid lines were more pronounced under ambient than elevated $\mathrm{CO}_{2}$.

\section{Discussion}

The occurrence and severity of drought is variable but rain-fed growing areas are particularly susceptible to yield losses from water deficiency. Up to $15 \%$ of the North American Corn Belt, which produces $40 \%$ of the global maize crop, experiences reduced yields annually due to drought. ${ }^{1}$ Future growing seasons also will experience episodes of severe drought, and the predicted effects of Global Climate Change will likely increase the frequency and duration of extreme weather events. ${ }^{3}$ Collectively, global food security will be challenged in the future and improving the stress tolerance of maize will be vital to reverse this trend.

\section{Biomass, water relations and gas exchange}

Drought tolerance in maize involves numerous complex processes that are under the control of a large and diverse set of genes. ${ }^{11} \mathrm{~A}$ well established root system that penetrates deeply into soils and improved silking and pollination are required to achieve high yields when maize plants are under stress. ${ }^{2,7}$ One response of plants to water stress is to promote root development but curtail shoot growth. ${ }^{12}$ This adaptive strategy decreases water consumption from the above ground foliage while maximizing the plant's ability to acquire water from the soil. In the current study, both the drought sensitive and intermediate hybrid lines exhibited up to $77 \%$ decreases in shoot growth in response to drought. The impact of drought on shoot growth of the drought tolerant maize hybrid was about half that of the other two hybrids and this was observed in both $\mathrm{CO}_{2}$ environments. Root DW of the intermediate and sensitive lines also was up to one third less in the water deficient compared to the water sufficient environments after 15 to $17 \mathrm{~d}$ of water stress in both $\mathrm{CO}_{2}$ treatments. In comparison, root DW of the tolerant maize hybrid line remained about the same when exposed to drought stress under ambient $\mathrm{CO}_{2}$ and increased by about one- fourth when plants were grown similarly under elevated $\mathrm{CO}_{2}$. Clearly, sustaining root growth and minimizing excess foliage development in response to water stress are important traits in maize that confer drought tolerance.As in our earlier study, ${ }^{9}$ drought negatively affected relative SWC, LWP, $\mathrm{P}_{\mathrm{n}}$ and $g_{s}$ and the impact of $\mathrm{CO}_{2}$ enrichment was to delay changes of these

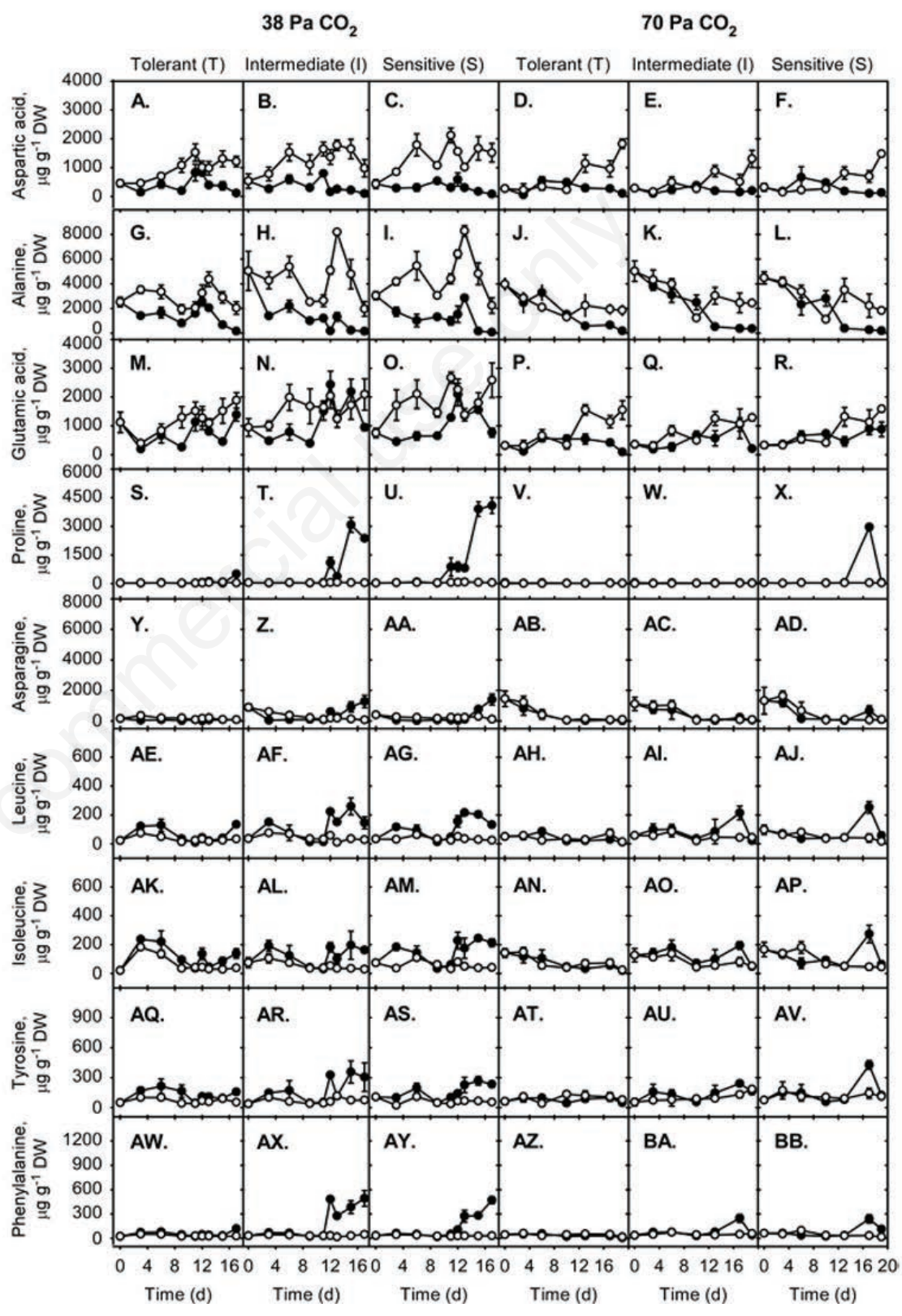

Figure 3. Effects of water stress and $\mathrm{CO}_{2}$ enrichment on soluble amino acids in leaves of three maize hybrids differing in drought susceptibility. Changes of nine individual amino acids $\left(\mu \mathrm{g} \mathrm{g}^{-1} \mathrm{DW}\right)$ in leaves of intermediate, tolerant and sensitive maize hybrids are shown during progressive drought treatment for $19 \mathrm{~d}$. Results are for water sufficient $(\circ)$ and water insufficient $(\bullet)$ plants raised in ambient (first three columns) or elevated (last three columns) $\mathrm{CO}_{2}$. Values are means \pm standard error for eight samples from two fully replicated experiments. 
three parameters by about $2 \mathrm{~d}$. Genotypic differences were observed for changes of SWC in this experiment. Rates of water consumption were greatest for the sensitive maize hybrid and were least for the drought tolerant hybrid. The minimum SWC for the tolerant maize hybrid line was about $25 \%$ of capacity and this occurred on the last measurement date. Therefore, the drought tolerant maize hybrid only experienced moderate water stress in this study and the extent of water stress experienced by the other two hybrid lines was comparatively more severe. It is likely that decreased foliage of the drought tolerant maize line required less water from the soil compared to the other two maize hybrid lines and this ultimately resulted in increased final SWC values. Low rates of soil water consumption due to lesser above ground growth by the drought tolerant maize hybrid also impacted various other parameters in this study. Overall, LWP and $\mathrm{P}_{\mathrm{N}}$ were affected similarly by drought in this study. Large decreases of LWP and $P_{N}$ in response to drought were observed for the sensitive maize line, whereas these two parameters in the tolerant maize line were less affected by drought. As mentioned above, minimum SWC values in the ambient and elevated $\mathrm{CO}_{2}$ treatments were similar for all three genotypes on the final harvest dates following 15 and 17 $\mathrm{d}$ of drought, respectively. Unlike SWC, final LWP and $\mathrm{P}_{\mathrm{N}}$ values of the intermediate and sensitive maize hybrids differed in the ambient and elevated $\mathrm{CO}_{2}$ treatments on the final harvest. It is clear that for these two genotypes $\mathrm{CO}_{2}$ enrichment mitigated the effects of drought on $\mathrm{P}_{\mathrm{N}}$ and LWP. Also, rates of PN of all three genotypes were roughly similar under well watered conditions. Therefore, differences in total and shoot biomass between the three genotypes in the water sufficient treatment were not the result of differing rates of $\mathrm{P}_{\mathrm{N}}$. Due to the effects of stomatal closure, ${ }^{13}$ doubling the ambient $\mathrm{CO}_{2}$ concentration of the well watered maize seedlings decreased measurements of $g_{s}$ for all three genotypes by about half. The relative effects of water stress on $g_{s}$ were decreased by $\mathrm{CO}_{2}$ enrichment for all of the genotypes used in this study. Consistent with changes of LWP, decreases of $g_{s}$ in response to drought in both $\mathrm{CO}_{2}$ environments were greatest for the sensitive hybrid line and were least for the tolerant hybrid line. The absolute accuracy of $g_{s}$ measurements in this study was potentially altered by non-uniform stomatal closure in response to water stress. ${ }^{13,14}$ However, the conclusion that $\mathrm{CO}_{2}$ enrichment mitigated the effects of water stress on $g_{s}$ of maize was confirmed (i.e., Downton et al.). ${ }^{15}$

\section{Soluble amino acids}

Changes of maize leaf soluble amino acids in response to the combined effects of water stress and $\mathrm{CO}_{2}$ enrichment have been described previously. ${ }^{9} 16$ There were 19 amines measured in this study and 11 of these increased in response to drought in the ambient $\mathrm{CO}_{2}$ treatment. All of the aromatic and branched chain amino acids were included in this group. One amino acid, Met, was unaffected by water stress in both $\mathrm{CO}_{2}$ treatments. In contrast to current findings, Witt et al. ${ }^{6}$ observed increased Met levels in maize leaves in response to drought. The other amines measured in this study, including Gly, Ser and Thr, had mixed responses to drought. Unlike our earlier study, ${ }^{9}$ consistent changes of Gly and Ser in response to drought were not observed in either $\mathrm{CO}_{2}$ treatment of the current study. This was likely because water stress treatments were applied more quickly in our earlier compared to the present study. None of the amino acids that accumulated in
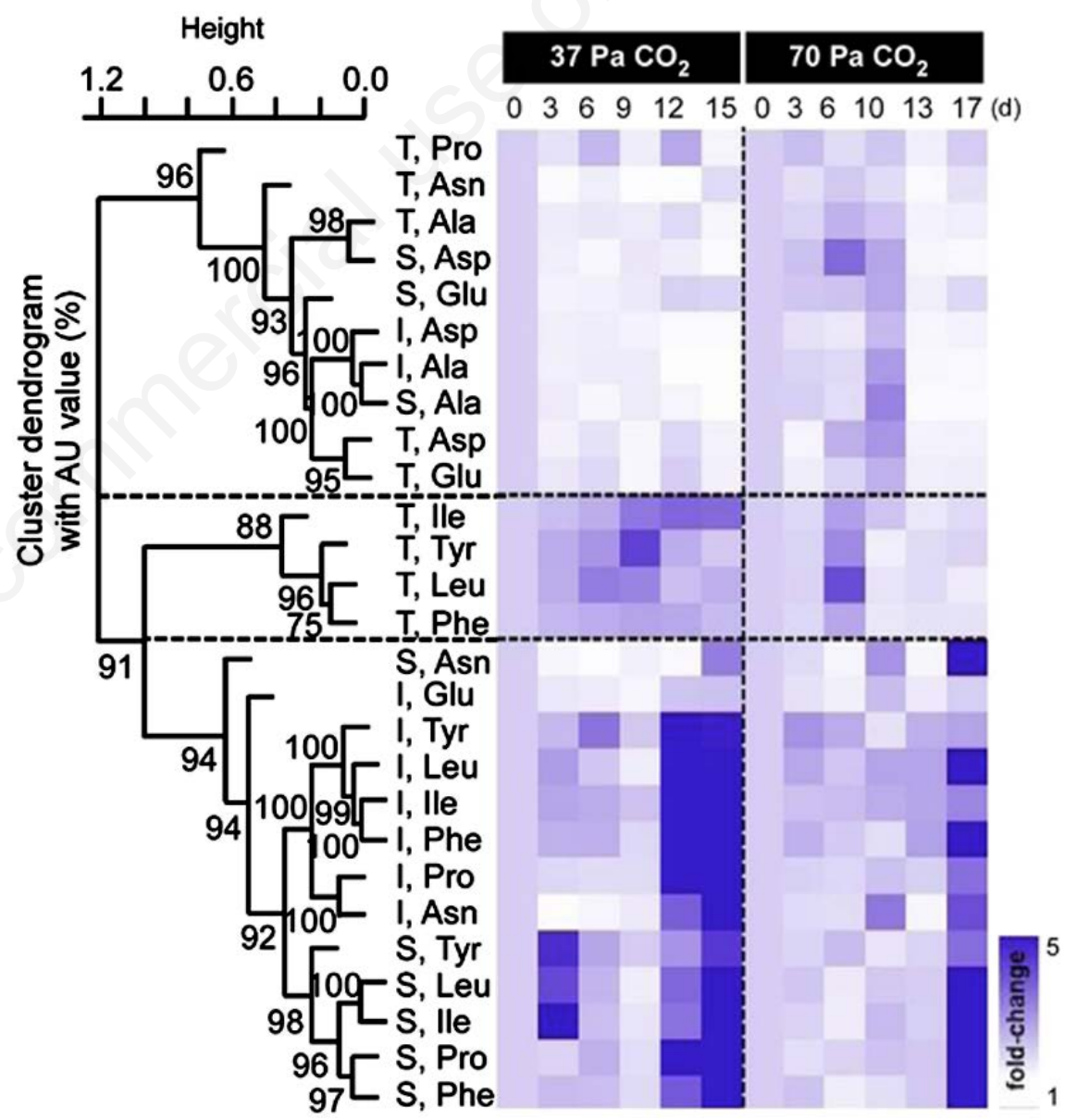

Figure 4. Hierarchical clustering analysis. Statistical relationships are shown for the combined effects of the duration of drought and $\mathrm{CO}_{2}$ enrichment on the 9 amino acids shown in Figure 3. Harvest dates corresponding to the duration of drought in either the ambient or elevated $\mathrm{CO}_{2}$ treatments were on the horizontal axis. Metabolite clusters depicting the relative differences between the water sufficient and insufficient samples are given on the vertical axis. Ratios that are 1:1 are shown in white and ratios greater than 1 are shown in gradations of blue. The abbreviations $T, I$ and $S$ refer to the tolerant, intermediate and sensitive maize hybrid lines, respectively. 
response to drought did so in the tolerant maize hybrid grown under elevated $\mathrm{CO}_{2}$. Changes of amino acids in the intermediate and sensitive genotype were small in the elevated compared to the ambient $\mathrm{CO}_{2}$ treatment and were only observed after 15 to $17 \mathrm{~d}$ of water stress treatment.

In agreement with previous results, ${ }^{9}$ Ala and Asp decreased almost to zero in all three genotypes when exposed to water stress and this occurred in both the ambient and elevated $\mathrm{CO} 2$ treatments. We concluded previously that decreases of Ala and Asp in maize leaves were impacted by inorganic nitrogen deficiency due to the water stress treatment. The finding that decreases of leaf Ala and Asp levels were similar in all three maize genotypes differing in drought tolerance supported this conclusion. In this study and previously, ${ }^{9}$ Glu decreased initially in response to mild water stress in the ambient $\mathrm{CO}_{2}$, although this temporarily reversed when water stress worsened. The accumulation of Pro in response to various stress treatments has been well studied in plants and Pro accumulation in various plant tissues has an important role in drought stress. ${ }^{17,18}$ In the current study, there was a 335 fold increase of Pro in response to drought in the sensitive maize hybrid under ambient $\mathrm{CO}_{2}$. The intermediate and drought tolerant maize hybrids accumulated less Pro than the sensitive maize hybrid in this study. This observation was also true for Phe. However, Leu, Ile and Tyr levels were as high or higher in the drought resistant maize hybrid than in the other two hybrids. Consequently, there were genotypic differences in changes of amino acids in response to drought in this study that were not directly proportional to changes of LWP and the extent of water stress.

Witt et $a l .{ }^{6}$ previously reported results of a greenhouse study using six maize hybrids differing in drought tolerance. The authors reported changes of over 100 metabolites in five different tissues in response to water stress that was imposed immediately before and during anthesis. This prior study identified several metabolites that were affected by water stress in maize leaves but genotypic differences in metabolite responses to drought were not detected. This was in direct contrast to the current study using amino acid results for leaf tissue of maize hybrids sampled during vegetative growth. Figure 4 shows that statistically significant genotypic differences occurred for the responses of amino acids to water stress and that it was easier to detect this in the ambient compared to the elevated $\mathrm{CO}_{2}$ treatment. The best metabolic indicator of genotypic variation in drought tolerance in the current study was Asn. In contrast to the drought tolerant hybrid, this compound accumulated in the intermediate and sensitive hybrid lines grown under ambient $\mathrm{CO}_{2}$. Also, Pro, Leu, Tyr and Phe began to accumulate after $10 \mathrm{~d}$ of drought treatment in leaves of the intermediate and sensitive maize hybrids. These same four amino acids began to accumulate after $15 \mathrm{~d}$ of drought treatment in the drought tolerant hybrid grown under ambient $\mathrm{CO}_{2}$. Therefore, genotypic differences in amino acid accumulation due to drought could be identified by carefully selecting harvesting times and treatment conditions.

\section{Conclusions}

In summary, our results demonstrated that maize genotypes selected for improved yields in water stressed environments differed from a control hybrid in several important ways during vegetative growth. Current findings suggested that improvements of drought tolerance in the most stress tolerant hybrid derived partly from decreased shoot growth under well watered treatment conditions. Drought tolerance of all three genotypes was improved by $\mathrm{CO}_{2}$ enrichment and elevated $\mathrm{CO}_{2}$ treatments ameliorated effects of low SWC on foliar water relations. Although there were important exceptions, increases of foliar amino acids in response to water stress were usually greater for the sensitive than the intermediate or tolerant maize hybrids. Conversely, amino acids that decreased in response to water stress changed independently of $\mathrm{CO}_{2}$ enrichment and of genotypic differences in LWP. Although we can not predict field performance from a controlled environment study, the drought tolerant maize genotype used in this study was very resistant to water stress based on all of the parameters tested.

\section{References}

1. Campos H, Cooper M, Habben JE, et al. Improving drought in maize: a view from industry. Field Crops Res 2004;90:19-34.

2. Bruce WB, Edmeades G0, Barker TC. Molecular and physiological approaches to maize improvement for drought tolerance. J Exp Bot 2002;53:13-25.

3. Sicher RC, Kim SH. Photosynthesis, growth and maize yields in the context of global change. In: Prioul JL, Thévenot C, Molnar T, eds. Advances in maize, essential reviews in experimental biology. London: Society for Experimental Biology; 2011. pp 373-391.

4. Tollefson J. Drought-tolerant maize gets US debut. Nature 2011;469:144.

5. La Rovere RG, Kostandini G, Abdoulaye T, et al. Potential impact of investments in drought tolerant maize in Africa. Adddis Ababa: IITA, CIMMYT; 2010.

6. Witt S, Galicia J, Cairns J, et al. Metabolic and phenotypic responses of greenhousegrown maize hybrids to experimentally controlled drought stress. Mol Plant 2011;1-18.

7. Araus JL, Serret MD, Edmeades G0. Phenotyping maize for adaptation to drought. Front Physiol 2012;3:1-20.

8. Harrigan GG, Ridley WP, Miller KD, et al. The forage and grain of MON 87460, a drought-tolerant corn hybrid, are compositionally equivalent to that of conventional corn. J Agric Food Chem 2009;57:9754-63.

9. Sicher RC, Barnaby JY. Impact of carbon dioxide enrichment on the responses of maize leaf transcripts and metabolites to water stress. Physiol Plant 2012;144:23853.

10. Suzuki R, Shimodaira H. Pvclust: an R package for assessing the certainty in the hierarchical clustering. Bioinformatics 2006;22:1540-2.

11. Cattivelli L, Rizza F, Badeck FW, et al. Drought tolerance in crop plants: an integrated view from breeding to genomics. Field Crops Res 2008;105:1-14.

12. Jefferies RA. Cultivar responses of water stress in potato: effects of shoot and roots. New Phytologist 1993;123:491-8.

13. Bunce J. Carbon dioxide effects on stomatal responses to the environment and water use by crops under field conditions. Oecologia 2004;140:1-10.

14. Leakey ADB, Uribelarrea M, Ainsworth EA, et al. Photosynthesis, productivity, and yield of maize are not affected by open-air elevation of $\mathrm{CO} 2$ concentration in the absence of drought. Plant Physiol 2006;140:779-90.

15. Downton WJS, Loveys BR, Grant WJ. Nonuniform stomatal closure induced by water stress causes putative non-stomatal inhibition of photosynthesis. New Phytologist 1988;110:503-9.

16. Foyer $\mathrm{CH}$, Valadier $\mathrm{MH}$, Mige A, Becker TW. Drought induced effects on nitrate reductase activity and mRNA and on the coordination of nitrogen and carbon metabolism in maize leaves. Plant Physiology 1998;117:283-92.

17. Delauney AJ, Verma DPS. Proline biosynthesis and osmoregulation in plants. Plant J 1993;4:215-23.

18. Sharp RE, Poroyko V, Hejlek LG, et al. Root growth maintenance during water deficits: physiology to functional genomics. J Exp Bot 2004;55:2343-51. 